\title{
Satellite Image Georeferencing on the Base of Coastlines' Fuzzy Masks
}

\author{
Liliya Demidova ${ }^{1,2}$ and Victor Ushenkin ${ }^{1, *}$ \\ ${ }^{1}$ Ryazan State Radio Engineering University, 390005, Ryazan, Russia \\ ${ }^{2}$ Moscow Technological Institute, 119334, Moscow, Russia
}

\begin{abstract}
The article discusses the possibility of using fuzzy masks in the problem of image georeferencing according to electronic maps. A new approach to membership assessment of the image fragment in fuzzy mask has been suggested. Fuzzy masks have been compared with binary masks by the accuracy of coregistration of image fragments and coastlines extracted from electronic maps. It's shown that fuzzy masks allow taking into account brightness characteristics of water and land in different regions of the Earth at different times of day and year. The results of experimental studies confirm the efficiency of the offered approach to image georeferencing based on the fuzzy masks.
\end{abstract}

\section{Introduction}

Inaccurate measurement of satellite angular orientation leads to significant errors in image georeferencing. It is especially typical of geostationary satellites having a great height of an orbit. To reduce these errors, it is necessary to coregister objects in the image and their corresponding referential objects.

In geometric processing of images from Russian geostationary satellite "Elektro-L" coastlines extracted from electronic maps are used as reference objects for precise georeferencing [1].

Referential coastlines are represented as binary masks, where land is coded by unit and water is coded by zero. An example of a binary mask is shown in Fig. 1. Pixels corresponding to water and land are shown in black and white respectively.

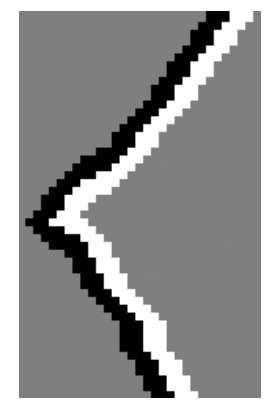

Fig. 1. An example of binary mask.

Coregistration of the image fragment and the binary mask is performed by maximizing the correlation function:

$$
\rho=\frac{\overline{Y_{1}}-\overline{Y_{0}}}{\sqrt{D}} \cdot \frac{\sqrt{q_{1} q_{0}}}{q_{1}+q_{0}},
$$

where $\bar{Y}_{1}$ and $\overline{Y_{0}}$ are average values of intensity in pixels under unit and zero parts of the binary mask, $D$ is variance of intensity in pixels under the mask, $q_{1}$ and $q_{0}$ are numbers of unit and zero values in the mask.

The accuracy and reliability of coregistration are negatively affected by such factors as the partial hiding of coastline in the image by clouds, incomplete conformity of coastlines in the image and electronic map. Therefore, it's necessary to develop alternative approaches to precise georeferencing using reference coastlines.

In the recent years, the theory of fuzzy sets is increasingly used in solving various applied problems, including image processing.

For the problem of precise image georeferencing we propose to replace binary masks of coastlines by fuzzy ones. Our purpose is to research the applicability of fuzzy masks, generated according to the shape of coastlines and brightness characteristics of water and land, in the problem of precise georeferencing.

\section{Theoretical part}

Fuzzy masks were first proposed by F. Russo and G. Ramponi [2]. They are meant to detect objects in the images.

To generate fuzzy mask, objects located in analyzed image fragment are classified. Each class is associated with a fuzzy set, its membership function is defined in the pixel brightness domain. The value of fuzzy set membership function is considered as the degree of truth of fuzzy logic statement "an object of this class is located in that pixel".

Fuzzy mask defines the relative positions of objects of different classes in the image fragment. Membership 
of the image fragment in the fuzzy mask is defined as conjunction of fuzzy logic statements considered above. It should be noted that generally (depending on the problem) a fuzzy mask can have any form and size.

B. Solaiman, R. Fiset and F. Cavayas suggested using fuzzy masks in the problem of road recognition [3]. Herewith, the ideas of the fuzzy set theory are used in many applied problems [4-9].

An example of the fuzzy mask by size of $3 \times 3$, describing a curved fragment of the road, is presented in Fig. 2. Pixels corresponding to the classes "Road" and "Surrounding background" are shown in white and gray respectively. The fuzzy sets associated with these classes have membership functions presented in Fig. 3.

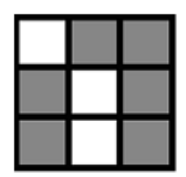

Fig. 2. An example of the fuzzy mask by size $3 \times 3$.

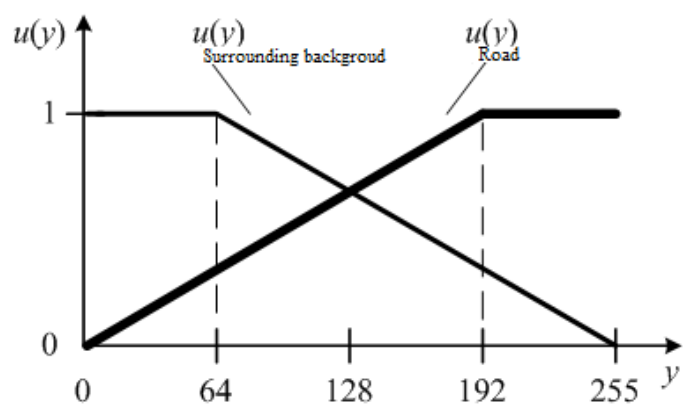

Fig. 3. The membership functions of fuzzy sets "Road" and "Surrounding background" are defined in the pixel brightness domain

To solve the problem of precise image georeferencing using coastlines fuzzy masks it is necessary to introduce the classes "Water" and "Land" into consideration. Thus, the fuzzy mask $M$ defining some coastline consists of $n$ pixels corresponding to one of the two introduced classes.

Membership of the image fragment $Y$ in the fuzzy mask $M$ is defined as

$$
u_{M}(Y)=\bigwedge_{i=1}^{n} u_{c_{i}}\left(y_{i}\right) \text {. }
$$

where $y_{i}$ is the brightness of image pixel under the $i$ th pixel of the fuzzy mask, $c_{i}$ is the class corresponding to the $i$ th pixel of the fuzzy mask, $u_{c}(y)$ is the membership function of the fuzzy set associated with the class $c, \wedge$ is the fuzzy conjunction operator.

Fuzzy conjunction may be implemented according to some $T$-norm.

A $T$-norm is a function $T:[0,1] \times[0,1] \rightarrow[0,1]$, which satisfies the following properties for any $x, y, z, w \in[0,1]$ :

- commutativity: $T(x, y)=T(y, x)$;

- monotonicity: $T(x, y) \leq T(z, w)$ if $x \leq z$ and $y \leq w$;
- associativity: $T(x, T(y, z))=T(T(x, y), z)$.

Besides, the boundary conditions are satisfied: $T(0,0)=T(0,1)=T(1,0)=0$ and $T(x, 1)=x(\forall x \in[0,1])$.

The following $T$-norms are most widespread:

$$
\begin{aligned}
& T_{1}(x, y)=\min (x, y) \text { and } T_{2}(x, y)=x y \\
& ((x, y) \in[0,1] \times[0,1]) .
\end{aligned}
$$

According to these $T$-norms, a fuzzy conjunction operator may be defined as

$$
u_{A \cap B}(y)=u_{A}(y) \wedge u_{B}(y)=\min \left(u_{A}(y), u_{B}(y)\right)
$$

or

$$
u_{A \cap B}(y)=u_{A}(y) \wedge u_{B}(y)=u_{A}(y) u_{B}(y),
$$

where $A$ and $B$ are the fuzzy sets with the membership functions $u_{A}(y)$ and $u_{B}(y)$ respectively.

The expression (3) was used by B. Solaiman, R. Fiset and F. Cavayas to evaluate membership of image fragment in a fuzzy mask under solving the problem of road recognition. However, the use of the expression (4) is more preferable because it's steadier against the noise.

For example, $(n-1)$ pixels of the image fragment have great (close to unit) values of $u_{c_{i}}\left(y_{i}\right)$ and one pixel contains the noise $\left(u_{c_{i}}\left(y_{i}\right)\right.$ is close to zero).

If the expression (3) is used, all $(n-1)$ "good" pixels are ignored and this leads to an erroneous conclusion that the image fragment doesn't correspond to the mask.

At the same time, the use of the expression (4) allows considering all $n$ values of $u_{c_{i}}\left(y_{i}\right)$. If most of them are close to unit it's concluded that the image fragment corresponds to the mask.

It also requires all values of $u_{c_{i}}\left(y_{i}\right)$ to be equal to zero. Thus, the membership function of each fuzzy set should be specified so that its minimum value is greater than zero. The minimum value of the membership function $u_{c_{i}}\left(y_{i}\right)$ is chosen in accordance with the probability of having noise in image pixel.

Taking use of the expression (4) into account, membership of the image fragment in the fuzzy mask can be rewritten as

$$
u_{M}(Y)=\prod_{i=1}^{n} u_{c_{i}}\left(y_{i}\right)
$$

The flaw in the expression (5) is that value of $u_{M}(Y)$ is very close to zero in the case of great $n$ and unideal compliance of the image fragment and the fuzzy mask. In such situation the result underflow can arise in machine calculations of the expression (5).

It is expedient to replace multiplication in the expression (5) with the geometric mean:

$$
u_{M}(Y)=\left(\prod_{i=1}^{n} u_{\mathrm{m}_{i}}\left(y_{i}\right)\right)^{\frac{1}{n}} .
$$

The value of $u_{M}(Y)$ defined as (6) always belongs to the range from $\min _{i=1, n}\left(u_{m_{i}}\left(y_{i}\right)\right)$ to $\max _{i=1, n}\left(u_{m_{i}}\left(y_{i}\right)\right)$. Moreover, the result of the comparison of $u_{M}\left(Y_{1}\right)$ and $u_{M}\left(Y_{2}\right)$ 
which are calculated using the expression (6) is the same that in the case of calculation using the expression (5).

Coregistration of the image fragment and reference coastline may be performed by maximizing the function (6).

To manage the coregistration results, it's possible to change membership functions of fuzzy sets "Land" and "Water".

\section{The experimental studies}

Coastline fragments with various degrees of uniqueness have been used in the experimental studies. The images had various spatial resolution and represent the Earth's surface regions with various areas.

The cases of the partial hiding of coastline in the image by clouds, complete and incomplete conformity of coastline in the image were considered.

Values of intensity in pixels corresponding to water and land depend on geographic location and time of the day and vary from image to image. So, two different pairs of the membership functions of the fuzzy sets "Water" and "Land" shown in Fig. 4 (a, b) have been used in the experimental studies.

The minimum value of all membership functions equal to 0.01 has been chosen, that is the probability of noisy pixels in the images has been estimated to be 0.01 .

Coregistration of coastline in the image and reference one is performed by maximizing the correlation function (1) with usage of binary mask, by maximizing the membership function (6) with the usage of a fuzzy mask, and by maximizing the geometric mean of (1) and (6) with the usage of the binary and fuzzy mask combination.

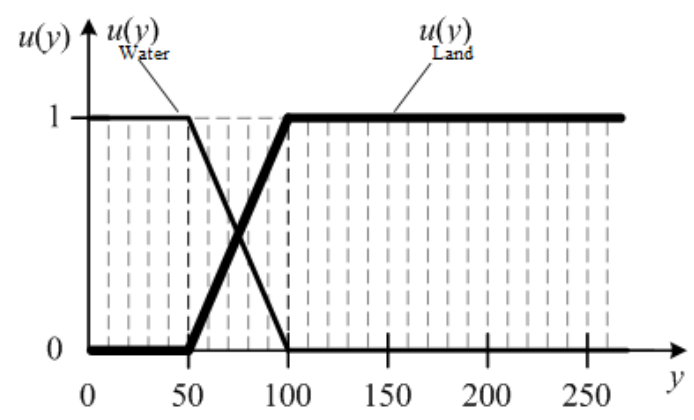

a

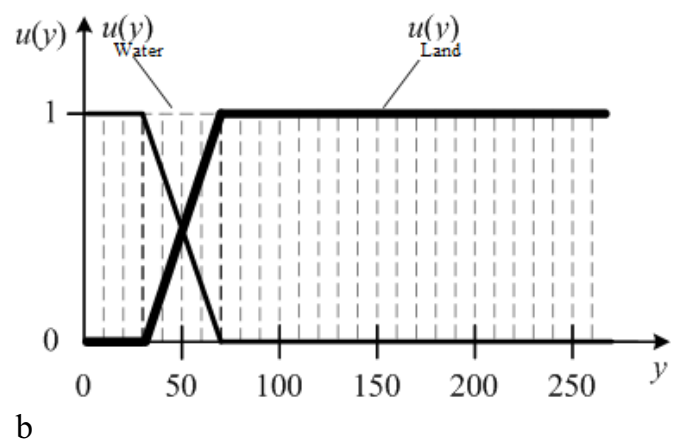

Fig. 4. The examples of the membership functions of the fuzzy sets "Water" and "Land"
Both fuzzy and binary masks provide the exact results at complete conformity of the mask and coastline in the image, good illumination and the lack of clouds.

These types of the masks may provide inaccurate results at low level of the reference coastline uniqueness because the probability of finding other similar borderline between light and dark objects becomes rather high.

Nevertheless, this probability is lower when using the fuzzy masks with thoroughly chosen membership functions of the fuzzy sets "Water" and "Land", than when using the binary masks.

In case of incomplete conformity of the mask and coastline in the image both types of the masks provide rather not exact coregistration.

However, the maximum value of the membership function (6) is rather low in this case, while the maximum value of the correlation function (1) is as high as in case of complete conformity of the mask and coastline in the image. Therefore, it's possible to detect such cases when using fuzzy masks and it's not possible when using binary ones.

Both types of the masks, as a rule, provide incorrect coregistration in case of partial hiding of the coastline by clouds. The maximum values of the functions (1) and (6) are low, that allows detecting such cases and reject incorrect coregistration results. The results of the binary masks are tittle more accurate, however their accuracy is still not high enough.

Thus, the usage of fuzzy masks is preferable with low level of the referential coastline uniqueness and with incomplete conformity of the mask and coastline in the image. In other cases fuzzy and binary masks provide similar results. The combination of fuzzy and binary masks provides, as a rule, more accurate coregistration, than the usage of each mask type separately, but requires more computational resources.

It should be taken into consideration, that some features of georeferencing are based on the fuzzy masks.

The accuracy of georeferencing depends on the choice of the fuzzy sets "Water" and "Land" membership functions.

It's necessary to take into consideration the land brightness characteristics in each region. So, for example, the surface of the Hindustan peninsula is significantly darker in the visible spectral range, than the surface of Africa. Besides, illumination of the land surface depends on time of the day and season. In this regard it is expedient to set individual membership functions to each of these cases, using known aprioristic data.

\section{Conclusions}

The results of the experiments allowed to make following conclusions. The approach to precise image georeferencing based on coregistration of image fragments and reference coastlines represented as fuzzy masks can be applied in practice.

The use of the formula (6) for membership function's evaluation of the image fragment to the fuzzy mask 
allows to exclude the possibility of the result overflow. If the restrictions on the performance time of georeferencing are not rigid, it's better to perform the coregistration using the combination of binary and fuzzy masks by maximizing the geometric mean of the functions (1) and (6).

In case of rigid restrictions on the performance time of georeferencing it is possible to use low value of the fuzzy mask membership function as additional rejection criterion of the binary mask and the image fragment coregistration result.

To receive correct results of image georeferencing using fuzzy masks it's necessary first to analyze brightness characteristics of water and land during various times of the day in various regions of the Earth and create database of fuzzy masks membership functions.

It should be noted that fuzzy masks won't have advantage in comparison with binary masks, if the same membership functions are used to all times of the day and all regions of the Earth.

\section{References}

1. N.A. Egoshkin, V.V. Eremeev et al., Current problems in remote sensing of the earth from space, 6, 132-138 (2009). [In Rus]
2. F. Russo, G. Ramponi, Working on Image Data Using Fuzzy Rules, Proc. Sixth European Signal Processing Conf. EUSIPCO-92. Bruxelles. pp. 1413-1416 (1992)

3. B. Solaiman, R. Fiset, F. Cavayas, IEEE International Geoscience and Remote Sensing Symposium Proceeding, pp. 894-896 (1998)

4. L.A. Demidova, N.I. Nesterov, Fuzzy and possibilistic segmentation of earth surface images by means of intelligent information technologies. Proceedings 2014 International Conference on Computer Technologies in Physical and Engineering Applications, ICCTPEA 2014, pp. 3536 (2014)

5. L.A. Demidova, Automation and Remote Control, 74, 313-320 (2013)

6. L.A. Demidova, S. V. Truhanov, Cloud of Science, 2, 40-60 (2015) [In Rus]

7. L. Demidova, Yu. Sokolova, E. Nikulchev, International Review on Modelling and Simulations, 8, 446-457 (2015)

8. L. Demidova, S. Trukhanov, Contemporary Engineering Sciences, 8, 885-900 (2015)

9. D. Van der Weken, M. Nachtegael, E.E. Kerre, Proceedings of IEEE Int. Conf. Acoustics, Speech and Signal Processing, pp. 3317-3320 (2002) 